\title{
Açáo de extrato e óleo de nim no controle de Rhipicephalus (Boophilus) microplus (Canestrini, 1887) (Acari: Ixodidae) em laboratório
}

\author{
Action of extract and oil neem in the control of Rhipicephalus (Boophilus) microplus \\ (Canestrini, 1887) (Acari: Ixodidae) in laboratory \\ Sônia Maria Forti Broglio-Micheletti*; Nivia da Silva Dias; Ellen Carine Neves Valente; Leilianne Alves de Souza; \\ Diego Olympio Peixoto Lopes; Jakeline Maria dos Santos
}

Laboratório de Entomologia, Centro de Ciências Agrárias - CECA, Universidade Federal de Alagoas - UFAL

Recebido em 14 de Abril de 2009

Aceito em 22 de Julho de 2009

\section{Resumo}

Extratos vegetais orgânicos e óleos emulsionáveis de Azadirachta indica A. Juss (Meliaceae) (nim) foram estudados com o objetivo de avaliar seus efeitos no controle de fêmeas ingurgitadas de Rhipicephalus (Boophilus) microplus (Canestrini, 1887) em laboratório. Foram utilizados extratos orgânicos hexânicos e alcoólicos a 2\% (peso/volume), em testes de imersão, durante 5 minutos, preparados com sementes, solubilizados em dimetilsulfóxido (DMSO) a 1\%. O experimento foi inteiramente casualizado, sendo constituído por 6 tratamentos e 5 repetiçóes, cada uma delas representada por 5 carrapatos. O grupo controle consistiu de fêmeas sem tratamento. Com base nos resultados deste trabalho, pode-se indicar que os tratamentos extrato de semente (hexano) e óleo emulsionável $\mathrm{I}^{1}$, em concentraçáo a 2\%, possuem significativo potencial adjuvante de controle do carrapato bovino, pois ocasionam a mortalidade nos primeiros dias após o tratamento e interferem na reproduçáo, mostrando ser uma alternativa aos carrapaticidas normalmente utilizados.

Palavras-chave: nim, Azadirachta indica, Rhipicephalus (Boophilus) microplus, carrapato, fitoterápicos.

\begin{abstract}
Organic plant extracts and emulsified oil of Azadirachta indica A. Juss (Meliaceae) (neem) were studied to evaluate its effects in control of engorged females of Rhipicephalus (Boophilus) microplus (Canestrini, 1887) in the laboratory. Hexane and alcoholic organic extracts, $2 \%$ (weight/volume) were used in tests of immersion for 5 minutes, prepared with seeds, solubilized in dimethylsulfoxide (DMSO) to $1 \%$. The experiment was entirely randomized, consisting of 6 treatments and 5 replicates, each represented by 5 ticks. Control groups consisted of untreated females. Based on the results of this work, we can indicate that the seed extract (hexanic fraction) and óleo emulsionável ${ }^{1}$ concentration to $2 \%$ have significant adjuvant potential to control the cattle tick, because, cause the mortality in the first days after the treatment and interfere in the reproduction, showing to be an alternative to acaricides normally used.
\end{abstract}

Keywords: neem, Azadirachta indica, Rhipicephalus (Boophilus) microplus, tick, phytoterapics.

${ }^{1}$ Base $\mathrm{Nim}^{\circ}$, Riomudas.

\section{Introdução}

O carrapato Rhipicephalus (Boophilus) microplus (Canestrini, 1887) (Acari: Ixodidae) é o mais importante ectoparasito em áreas de exploração pecuária, tanto em regiôes tropicais quanto subtropicais, sendo responsável por severas perdas econômicas (ATHAYDE et al., 2001). Essa espécie, além de causar espoliação sanguínea em virtude do hematofagismo, lesa o couro e é o

\footnotetext{
*Autor para correspondência: Sônia Maria Forti Broglio-Micheletti

Professora, Laboratório de Entomologia, Centro de Ciências Agrárias - CECA, Universidade Federal de Alagoas - UFAL, Campus Delza Gitaí,

Rodovia BR 104, km 85, s/n, CEP 57100-000 Rio Largo - AL, Brasil

e-mail: soniamfbroglio@gmail.com

Apoio: Sob os auspícios da FINEP/SECT/FAPEAL/CNPq.
}

principal transmissor de agentes patogênicos para os bovinos (BITTENCOURT et al., 1999).

O controle do R. (B.) microplus é feito principalmente por meio de produtos químicos, aplicados sobre o animal, com o objetivo de combater as fases parasitárias do carrapato (SANTOS JÚNIOR; FURLONG; DAEMON, 2000). O uso incorreto do carrapaticida (subdoses, preparos inadequados, aplicaçóes mal realizadas, etc.) faz com que os carrapatos não morram após contato com o produto. Cada vez que os carrapatos sobrevivem a uma aplicação de carrapaticida, eles transmitem às geraçôes posteriores informaçôes genéticas de como sobreviver àquele produto (FURLONG; PRATA, 2006). 
Dessa forma, o quadro atual do controle químico do carrapato caracteriza-se pelo aumento progressivo dos casos de resistência desse ectoparasito e, consequentemente, pelo aumento na frequência da aplicação de acaricidas, com a presença de resíduos desses produtos no leite e na carne (MENDES et al., 2007). Assim, o combate ao carrapato está direcionado a pesquisas de estratégias de controle, isto é, uma combinação do uso prudente e racional dos parasiticidas disponíveis com as alternativas de controle, que levam à manutenção de populaçóes parasitárias abaixo do seu limiar econômico com um mínimo impacto ambiental (FAO, 2003). Como métodos alternativos não-químicos, incluem-se o uso de animais geneticamente resistentes, o desenvolvimento de vacinas, o gerenciamento de pastagem com alternância de espécies e o controle biológico (LEAL; FREITAS; VAZ Jr., 2003).

A necessidade de métodos mais seguros, menos agressivos ao homem e ao meio ambiente, tem estimulado a busca de novos acaricidas a partir de extratos vegetais. Dessa forma, acredita-se que o uso de extratos vegetais de uma forma isolada ou associada pode causar um desenvolvimento bem mais lento da resistência. Outro fator importante é a redução do problema de resíduos bem como sua característica biodegradável (MORALES; GARCÍA, 2000; ROEL, 2001; IANNACONE; LAMAS, 2002).

A utilização de extratos vegetais no controle do carrapato tem sido foco de pesquisas em vários países (CHUNGSAMARNYART et al., 1991; WILLIAMS, 1993; VATSYA et al., 2006; ÁLVAREZ et al., 2008). No Brasil, trabalhos que utilizaram óleos emulsionáveis de eucalipto (Eucalyptus spp.) (Myrtaceae), rotenoides extraídos do timbó (Derris urucu) (Fabaceae) (VERÍSSIMO, 2004), e azadirachtina, presente em plantas da família Meliaceae (Melia azedarach) (BORGES et al., 2003; SOUSA et al., 2008), mostraram-se promissores no controle desse parasito.

As plantas da família Meliaceae são as mais exploradas por possuírem compostos secundários, que são encontrados em todas as partes da planta, principalmente nas folhas, frutos e sementes. $\mathrm{O}$ nim, Azadirachta indica A. Juss, é a espécie botânica atualmente mais estudada e classificada como um pesticida de alta eficiência e baixo efeito residual (MARTINEZ, 2002; AGUIAR-MENEZES, 2005). O princípio ativo azadirachtina, contido no nim, pode tornar-se importante no controle de pragas, pois tem largo espectro de ação, é compatível com outras formas de manejo, não tem açáo fitotóxica, é praticamente atóxica ao homem e não agride o meio ambiente (MARTINEZ, 2002). Extratos dessa planta foram utilizados para o controle de algumas espécies de carrapatos como Hyalomma anatolicum excavatum Koch (Acarina: Ixodidae), Amblyomma americanum L. (Acarina: Ixodidae) e Dermacentor variabilis Say (Acarina: Ixodidae) (ABDEL-SHAFY; ZAYED, 2002; SANTOS et al., 2006).

Dentre as várias marcas de nim comercializadas como óleo emulsionável, não se tem a informação precisa de quais substâncias estão contidas no produto final e sua eficácia no controle do carrapato bovino.

O presente trabalho teve como objetivo estudar, em laboratório, a mortalidade (\%) e o tempo letal (dias) em fêmeas ingurgitadas de $R$. (B.) microplus submetidas a extratos e óleos emulsionáveis de nim e solvente (DMSO), em testes de imersão, bem como o número de massas de ovos oriundas dessas fêmeas, peso da massa de ovos (g), eclosão larval (\%) e eficiência do controle (\%).

\section{Material e Métodos}

Os bioensaios foram conduzidos no Laboratório de Entomologia do Centro de Ciências Agrárias da Universidade Federal de Alagoas (CECA, UFAL), localizado no município de Rio Largo, $\mathrm{AL}\left(9^{\circ} 27^{\prime} \mathrm{S}\right.$ e $35^{\circ} 27^{\prime} \mathrm{W}$, altitude 127 metros), à temperatura média de $27,6 \pm 1{ }^{\circ} \mathrm{C}$ e umidade relativa média de $77,4 \%$, em novembro e dezembro de 2008.

Foram utilizados óleos emulsionáveis de nim em duas marcas comerciais (I e II) ${ }^{1}$ adquiridas no comércio local e extratos de sementes da mesma planta preparados no Laboratório de Produtos Naturais, do Instituto de Química e Biotecnologia da Universidade Federal de Alagoas (UFAL).

Após a coleta das sementes, foi realizada a secagem em estufa com circulação e renovaçáo de ar a $40-45^{\circ} \mathrm{C}$, durante 48 horas e, em seguida, fez-se a trituração em moinho de facas para obtenção de pó fino, o qual foi imerso em hexano, na proporçáo de 1 parte de pó para 3 partes do solvente, durante 96 horas. $\mathrm{O}$ extrato foi filtrado, e a suspensão e a evaporação do solvente realizada em evaporador rotativo a vácuo $\left(50{ }^{\circ} \mathrm{C}\right.$, à pressáo reduzida) e, posteriormente, sobre a torta restante foi realizada mais uma extração com etanol. Os resíduos concentrados obtidos na extração foram dissolvidos em etanol, colocados em frascos de vidro previamente etiquetados e acondicionados em capela para a evaporação máxima do solvente. Para a obtençáo dos extratos brutos, cada um deles foi submetido ao processo de liofilização. Um dia antes, o material foi colocado em freezer para, em seguida, ser liofilizado por 24 horas. Dessa forma, obteve-se o extrato bruto, sem solvente e água. $\mathrm{Na}$ etapa seguinte, cada extrato bruto foi pesado com o frasco para o ajuste do volume extraído. Esses frascos foram acondicionados em geladeira, a $\pm 5^{\circ} \mathrm{C}$, para evitar a ocorrência de microrganismos.

As fêmeas ingurgitadas de $R$. (B.) microplus foram coletadas de animais isentos de carrapaticida químico de contato por pelo menos 30 dias. Os locais de coleta nos bovinos foram o úbere, tetos, testículos, entrepernas, papeira e o pavilhão auricular, que são as estruturas em que frequentemente são encontradas (GONZALES, 1975; CORDOVÉS, 1997), tendo-se o cuidado de separar fêmeas maiores que $5 \mathrm{~mm}$ em comprimento. As fêmeas ingurgitadas coletadas foram acondicionadas em placas de Petri e levadas ao laboratório em caixa térmica contendo gelo, para evitar oviposiçáo prematura e reduzir a mobilidade do parasito dentro das placas. Posteriormente, foram pesadas em balança analítica BG 400 (Quimis ${ }^{\circledR}$ ), com precisão de 0,001 g.

O delineamento experimental foi inteiramente casualizado com seis tratamentos e cinco repetiçóes, considerando-se cinco fêmeas ingurgitadas como repetição. O grupo controle foi representado por fêmeas isentas de quaisquer tratamentos. Os tratamentos foram compostos pelos extratos orgânicos (hexânico e etanólico) e óleos emulsionáveis I e II, 2\% (peso/volume), os quais foram, no momento da montagem do bioensaio, solubilizados em dimetilsulfóxido (DMSO) a 1\% e diluídos em água destilada e esterilizada, formando assim soluçóes de $100 \mathrm{~mL}$, nas quais 25 fêmeas foram

${ }^{1}$ I: Base Nim ${ }^{\oplus}$, Riomudas, http://www.riomudas.com.br/oleo_nim.htm; II: Nim-I-Go ${ }^{\oplus}$, Agrobiológica Soluçōes Naturais, http://www.organicsnet.com.br/ produto_detalhe.php?id_prod=502\&busca= 
imersas durante 5 minutos. Visando à avaliação da sensibilidade das fêmeas ao solvente DMSO, incluiu-se o tratamento água destilada e esterilizada em que se adicionou DMSO 1\%. Todo o material biológico permaneceu em condiçôes ambientais.

As coletas de dados foram feitas a partir do terceiro dia da montagem do bioensaio, onde foram registradas, a cada três e quatro dias até as primeiras eclosóes larvais, a mortalidade das fêmeas, número de oviposiçóes, peso da massa de ovos e percentual de eclodibilidade (14 dias após a última pesagem da massa de ovos) por meio de porcentagens, com variação de 0 a $100 \%$, eficiência do produto e tempo letal. Os cálculos para eficiência do produto (EP) foram avaliados segundo as equaçóes prescritas por DRUMMOND et al. (1973). Os valores obtidos foram submetidos à análise de variância, sendo as médias comparadas pelo teste de Tukey a $5 \%$ de probabilidade.

\section{Resultados e Discussão}

A mortalidade variou de $20 \pm 6,4 \%$ a $96 \pm 4,0 \%$, alcançando valor estatisticamente igual e superior para óleo emulsionável I e extrato da semente (hexano), que foram os tratamentos mais promissores e diferentes do óleo emulsionável II. Os tratamentos: extrato da semente (etanol), controle e água + DMSO foram iguais entre si, apresentando valores de mortalidade, respectivamente, $32 \pm 8,4 \%, 24 \pm 7,4 \%$ e $20 \pm 6,4 \%$ (Tabela 1). Esses resultados divergem dos obtidos por Oliveira et al. (2007), que concluíram que somente a utilização de soluçóes preparadas com óleo comercial de nim, enriquecidas em teor de azadirachtina-A por meio da maceração de sementes trituradas em hexano e metanol, com concentraçóes acima de $25 \%$, poderão ser eficientes para eliminar as fêmeas.

Quanto à sensibilidade ao DMSO, Chagas et al. (2003), relataram como excelente permeabilizador de membranas, permitindo a penetração do produto a ser testado de maneira rápida; no entanto, o fato de causar alta mortalidade em $R$. (B.) microplus prejudica seu uso, mesmo em concentraçáo de $25 \%$.

Em relação à oviposição, os tratamentos extrato da semente (hexano) e óleo emulsionável I mostraram ter grande potencial na redução do número de massas de ovos $(11,00 \pm 2,92$ e 12,20 $\pm 1,28$, respectivamente), diferindo dos demais tratamentos, que foram iguais entre si, variando entre $21,00 \pm 1,05$ a $22,80 \pm 0,92$. Extrato da semente (hexano) e óleo emulsionável I tiveram, respectivamente, $0,20 \pm 0,07 \mathrm{~g}$ e $0,23 \pm 0,01 \mathrm{~g}$, menores pesos de massas de ovos, com os demais tratamentos não diferindo entre si (variação entre $0,39 \pm 0,04 \mathrm{~g}$ a $0,46 \pm 0,05 \mathrm{~g})$ (Tabela 1$)$.

Tabela 1. Porcentagem \pm (erro-padrão da média) da mortalidade das fêmeas ingurgitadas, número de oviposiçóes, peso da massa de ovos, porcentagem de eclosão larval, e porcentagem da eficiência do produto em Rhipicephalus (Boophilus) microplus (Canestrini, 1887), após a utilização de extratos da semente e óleos emulsionáveis a base de nim a 2\% (peso/volume); água + DMSO 1\% e do grupo controle em teste in vitro, com 25 fêmeas/tratamento.

\begin{tabular}{|c|c|c|c|c|c|}
\hline Tratamento & $\begin{array}{c}\text { Mortalidade } \\
(\%)\end{array}$ & No de oviposiçóes & $\begin{array}{c}\text { Peso da massa de ovos } \\
(\mathrm{g})\end{array}$ & $\begin{array}{c}\text { Eclosáo } \\
(\%)\end{array}$ & $\begin{array}{c}\text { Eficiência do produto } \\
(\%)\end{array}$ \\
\hline Controle & $24,00 \pm 7,4 a b$ & $22,60 \pm 1,86 b$ & $0,46 \pm 0,05 c$ & $100,00 \pm 0,00 \mathrm{a}$ & $0,00 \pm 0,00 \mathrm{a}$ \\
\hline Água + $\mathrm{DMSO}^{1}$ & $20,00 \pm 6,4 a$ & $22,80 \pm 0,92 b$ & $0,45 \pm 0,03 c$ & $75,00 \pm 0,00 \mathrm{a}$ & $21,42 \pm 11,24 b$ \\
\hline Óleo emulsionável I² & $96,00 \pm 4,0 \mathrm{c}$ & $12,20 \pm 1,28 a$ & $0,23 \pm 0,01 \mathrm{ab}$ & $80,00 \pm 9,35 \mathrm{a}$ & $65,60 \pm 11,01 \mathrm{c}$ \\
\hline Óleo emulsionável II³ & $48,00 \pm 8,0 b$ & $21,00 \pm 1,05 b$ & $0,39 \pm 0,04 b c$ & $100,00 \pm 0,00 \mathrm{a}$ & $17,60 \pm 8,18 \mathrm{ab}$ \\
\hline Extrato semente (hexano) & $96,00 \pm 4,0 \mathrm{c}$ & $11,00 \pm 2,92 \mathrm{a}$ & $0,20 \pm 0,07 \mathrm{a}$ & $40,00 \pm 16,96 b$ & $73,20 \pm 18,77 \mathrm{c}$ \\
\hline Extrato semente (etanol) & $32,00 \pm 8,4 \mathrm{ab}$ & $21,20 \pm 1,24 b$ & $0,43 \pm 0,02 c$ & $100,00 \pm 0,00 \mathrm{a}$ & $17,00 \pm 10,22 \mathrm{ab}$ \\
\hline C.V( $\%)$ & 21,83 & 23,02 & 20,18 & 59,35 & 26,40 \\
\hline
\end{tabular}

Valores seguidos pela mesma letra nas colunas năo diferem estatisticamente entre si pelo teste de Tukey a $5 \%$ de probabilidade $(\mathrm{p}<0,05)$.

${ }^{1}$ Dimetilsulfóxido.

${ }^{2}$ Base Nim ${ }^{\oplus}$, Riomudas, http://www.riomudas.com.br/oleo_nim.htm.

${ }^{3} \mathrm{Nim}-\mathrm{I}-\mathrm{Go}{ }^{\circ}$, Agrobiológica, http://www.organicsnet.com.br/produto_detalhe.php?id_prod=502\&busca.

Tabela 2. Valores acumulados (\%) de fêmeas ingurgitadas mortas no período de 4 a 21 dias após a imersão (DAI) em extratos da semente e óleos emulsionáveis a base de nim a $2 \%$ (peso/volume), água + DMSO 1\% e do grupo controle em teste in vitro, com 25 fêmeas/tratamento.

\begin{tabular}{lrccccc}
\hline \multicolumn{7}{c}{ DAI (Dias Após Imersáo) } \\
\hline Tratamentos & 4 & 7 & 11 & 14 & 18 & 21 \\
Controle & $0 \mathrm{a}$ & $0 \mathrm{a}$ & $0 \mathrm{a}$ & $8,00 \mathrm{~b}$ & $12,00 \mathrm{a}$ & $24,00 \mathrm{~b}$ \\
Água + DMSO $^{1}$ & $0 \mathrm{a}$ & $0 \mathrm{a}$ & $0 \mathrm{a}$ & $4,00 \mathrm{a}$ & $20,00 \mathrm{c}$ & $20,00 \mathrm{a}$ \\
Óleo emulsionável I $^{2}$ & $12,00 \mathrm{c}$ & $24,00 \mathrm{c}$ & $24,00 \mathrm{c}$ & $36,00 \mathrm{~d}$ & $88,00 \mathrm{~d}$ & $96,00 \mathrm{e}$ \\
Óleo emulsionável II $^{3}$ & $0 \mathrm{a}$ & $8,00 \mathrm{~b}$ & $12,00 \mathrm{~b}$ & $12,00 \mathrm{c}$ & $16,00 \mathrm{~b}$ & $48,00 \mathrm{~d}$ \\
Extrato semente (hexano) $_{\text {Extrato semente (etanol) }}^{28,00 \mathrm{~d}}$ & $32,00 \mathrm{~d}$ & $44,00 \mathrm{~d}$ & $60,00 \mathrm{e}$ & $88,00 \mathrm{~d}$ & $96,00 \mathrm{e}$ \\
\hline
\end{tabular}

Valores seguidos pela mesma letra nas colunas năo diferem estatisticamente entre si pelo teste de Tukey a $5 \%$ de probabilidade $(\mathrm{p}<0,05)$.

${ }^{1}$ Dimetilsulfóxido.

${ }^{2}$ Base Nim ${ }^{\circ}$, Riomudas, http://www.riomudas.com.br/oleo_nim.htm.

${ }^{3} \mathrm{Nim}-\mathrm{I}-\mathrm{Go}^{\circ}$, Agrobiológica, http://www.organicsnet.com.br/produto_detalhe.php?id_prod=502\&busca. 
Esses resultados estão parcialmente em concordância com os obtidos por Borges et al. (2003), que utilizaram Melia azedarach (Meliaceae) a 0,25\% observando in vitro inibição total da oviposição em fêmeas ingurgitadas imersas no extrato bruto de frutos maduros extraídos com diferentes solventes. Além disso, observaram elevada taxa de mortalidade de larvas e alta eficácia sobre fêmeas ingurgitadas. Embora o extrato não tenha matado as fêmeas adultas, inibiu total ou parcialmente a produção de ovos e a embriogênese, diferentemente do que se observou, pois a mortalidade de fêmeas nesse bioensaio foi elevada.

O extrato da semente (hexano) foi o único que diferiu estatisticamente dos demais tratamentos em relação à eclosão larval $(40,00 \pm 16,96 \%)$, sendo a variação para os demais tratamentos entre 75,00 $\pm 0,00 \%$ e $100,00 \pm 0,00 \%$ (Tabela 1 ).

Analisando-se a eficiência dos extratos botânicos, observou-se que o nim (fração hexânica) foi o mais promissor $(73,20 \pm 18,77 \%$ ), sendo estatisticamente igual ao óleo emulsionável I (65,60 \pm 11,01\%). Os demais tratamentos mostraram-se iguais entre si, com valores entre $0 \%$ e 21,42 $\pm 11,24 \%$ (Tabela 1 ), com exceção de água + DMSO (21,42 + 11,24\%). Segundo Aguiar-Menezes (2005), a azadirachtina inibe a biossíntese do hormônio protoracicotrópico (PTTH) e, como consequência, não ocorre a biossíntese de outros hormônios, o que impossibilita os passos normais da troca de tegumento (ecdise) e também inibe a maturação dos ovos. Valente, Barranco e Sellaive-Villaroel (2007) compararam in vivo a eficácia do extrato aquoso de folhas frescas de nim $(1 \mathrm{~kg}$ de folhas frescas: $5 \mathrm{~L}$ de água), aplicado em banhos semanais (2 L/animal) durante um mês, com a da abamectina, em aplicação uma vez no dorso, para controle de $R$. $(B)$. microplus. Constataram que não houve diferença na infestação entre os grupos, podendo o extrato vegetal substituir a abamectina dentro de um programa zootécnico de controle dos carrapatos no semiárido.

Em relação ao tempo letal, os tratamentos controle e água + DMSO começaram a matar a partir dos 14 dias após a imersão (DAI). Em contrapartida, extrato da semente (hexano), óleo emulsionável I e extrato da semente (etanol) sobressaíram já aos 4 DAI, eliminando, respectivamente, 28, 12 e 4\% das fêmeas. A mortalidade foi se incrementando com o passar do tempo, alcançando aos 14 DAÍ, 60, 36 e 12\% das fêmeas mortas para extrato da semente (hexano), óleo emulsionável I e extrato da semente (etanol), respectivamente. Com 18 DAÍ, os tratamentos extrato da semente (hexano) e óleo emulsionável I se igualaram (88\% de fêmeas mortas), e, com 21 DAÍ, atingiram 96\%. O controle obteve no máximo $24 \%$ de fêmeas mortas e água + DMSO 20\% (Tabela 2).

No bioensaio realizado, apesar de ter vindo de mesma fonte (matéria-prima semente), o extrato da semente (hexano) possibilitou a extração direta da azadirachtina. Essa observação não ocorreu com o extrato da semente (etanol), que extraiu somente princípios ativos contidos na torta de nim.

Com base nos resultados deste trabalho, pode-se indicar que os tratamentos extrato da semente de nim (hexano) e óleo emulsionável I, em concentração a $2 \%$, possuem significativo potencial adjuvante no controle do carrapato bovino, pois, além de ocasionar a mortalidade das fêmeas ingurgitadas, nos primeiros dias após o tratamento, interferem na sua reprodução, mostrando ser uma alternativa aos carrapaticidas normalmente utilizados.

\section{Agradecimentos}

À FINEP/SECT/FAPEAL/CNPq pelo suporte financeiro à pesquisa e pelas bolsas de estudo.

\section{Referências}

ABDEL-SHAFY, S.; ZAYED, A. A. In vitro acaricidal effect of plant extract of neem seed oil (Azadirachta indica) on egg, immature, and adult stages of Hyalomma anatolicum excavatum (Ixodoidea: Ixodidae). Veterinary Parasitology, v. 106, n. 1, p. 89-96, 2002.

AGUIAR-MENEZES, E. L. Inseticidas Botânicos: seus princípios ativos, modo de ação e uso agrícola. Seropédica: Embrapa Agrobiologia, 2005. 58 p. (Documentos, 205).

ÁLVAREZ, V. et al. Control in vitro de garrapatas (Boophilus microplus; Acari: Ixodidae) mediante extractos vegetales. Revista de Biologia Tropical, v. 56, n. 1, p. 291-302, 2008.

ATHAYDE, A. C. R.; FERREIRA, U. L.; LIMA, E. A. L. A. Fungos entomopatogênicos: uma alternativa para o controle do carrapato bovino: Boophilus microplus. Biotecnologia Ciência e Desenvolvimento, v. 21, n. 1, p. 12-15, 2001.

BITTENCOURT, V. R. E. P.; MASCARENHAS, A. G.; FACCINI, J. L. H. Mecanismo de infecção do fungo Metarhizium anisopliae no carrapato Boophilus microplus em condições experimentais. Ciência Rural, v. 29, n. 2, p. 351-354, 1999.

BORGES, L. M. F. et al. In vitro efficacy of extracts of Melia azedarach against the tick Boophilus microplus. Medical and Veterinary Entomology, v. 17, n. 2, p. 228-231, 2003.

CHAGAS, A. C. S. et al. Sensibilidade do carrapato Boophilus microplus a solventes. Ciência Rural, v. 33, n. 1, p. 109-114, 2003.

CHUNGSAMARNYART, N.; JIWAJINDA, S.; JANSAWAN, W. Acaricidal effect of plant crude-extracts on the cattle tick (Boophilus microplus). Kasetsart Journal, v. 25, n. 5, p. 90-100, 1991.

CORDOVÉS, C. O. Carrapato: controle ou erradicaçấo. 2 ed. Guaíba: Agropecuária, 1997.176 p.

DRUMMOND, R. O. et al. Boophilus annulatus and B. microplus laboratory tests of insecticides. Journal of Economic Entomology, v. 66 , n. 1, p. 130-133, 1973 .

FOOD AND AGRICULTURE ORGANIZATION - FAO. Resistência a los antiparasitários: estado actual com énfasis em América Latina. Roma, 2003. 52 p. (Producción y Sanidad Animal).

FURLONG, J.; PRATA, M. Controle estratégico do carrapato dos bovinos de leite. Juiz de Fora: EMBRAPA, 2006. 2 p. (Circular Técnica, 38).

GONZALES, J. C. O controle do carrapato dos bovinos. Porto Alegre: Sulina, 1975. $103 \mathrm{p}$.

IANNACONE, J.; LAMAS, G. Efecto de dos extractos botánicos y un insecticida convencional sobre el depredador Chrysoperla externa. Manejo Integrado de Plagas y Agroecología, v. 65, p. 92-101, 2002.

SANTOS Jr., J. C. B.; FURLONG, J.; DAEMON, E. Controle do carrapato Boophilus microplus (Acari: Ixodidae) em sistemas de produção de leite da microrregião fisiográfica fluminense do Grande Rio-Rio de Janeiro. Ciência Rural, v. 30, n. 2, p. 305-311, 2000. 
LEAL, A. T.; FREITAS, D. R. J.; VAZ Jr., I. S. Perspectivas para o controle do carrapato bovino. Acta Scientiae Veterinariae, v. 31, n. 1, p. 1-11, 2003.

MARTINEZ, S. S. O Nim, Azadiractina indica: natureza, usos múltiplos, produção. Londrina: IAPAR, 2002. 142 p.

MENDES, M. C.; LIMA, C. K. P.; PRADO, A. P. Determinação da freqüência de realização de bioensaios para o monitoramento da resistência do carrapato Boophilus microplus (Acari: Ixodidae). Arquivos do Instituto Biológico, v. 74, n. 2, p. 87-93, 2007.

MORALES, S.; GARCÍA, C. M. Metodología para la evaluación del potencial insecticida de especies forestales. Revista Facultad Nacional Agronomía, v. 53, n. 1, p. 787-800, 2000.

OLIVEIRA, M. C. S. et al. Açáo de extrato de neem sobre Rhipicephalus (Boophilus) microplus com azadirachtina-A quantificada por HPLC. São Carlos: Embrapa, 2007. Disponível em: <http://www.repdigital.cnptia.embrapa.br/bitstream/CPPSE/17370/1/ PROCI(MCSO2007.00304).pdf >. Acesso em: 08 de fevereiro de 2009.

ROEL, A. R. Utilização de plantas com propriedades inseticidas: uma contribuição para o desenvolvimento rural sustentável. Revista Internacional de Desenvolvimento Local, v. 1, n. 2, p. 43-50, 2001.
SANTOS, A. C. G. et al. Uso de extrato de nim no controle de acaríase por Myobia musculi Schranck (Acari: Miobidae) e Myocoptes musculinus Koch (Acari: Listrophoridae) em Camundongos (Mus musculus var. albina L.). Neotropical Entomology, v. 35, n. 2, p. 269-272, 2006.

SOUSA, L. A. D. et al. Avaliação da eficácia de extratos oleosos de frutos verdes e maduros de cinamomo (Melia azedarach) sobre Rhipicephalus (Boophilus) microplus (Acari: Ixodidae). Revista Brasileira de Parasitologia Veterinária, v. 17, n. 1, p. 36-40, 2008.

VALENTE, M.; BARRANCO, A.; SELLAIVE-VILLAROEL, A. B. Eficácia do extrato aquoso de Azadirachta indica no controle de Boophilus microplus em bovino. Arquivo Brasileiro de Medicina Veterinária e Zootecnia, v. 59, n. 5, p. 1341-1343, 2007.

VATSYA, S. et al. In vitro acaricidal effect of some medicinal plantas against Boophilus microplus. Journal of Veterinary Parasitology, v. 20, n. 2, p. 141-143, 2006.

VERÍSSIMO, C. J. Controle biológico e alternativo do carrapato do boi. São Paulo: APTA, 2004. 3 p.

WILLIAMS, L. A. D. Adverse effects of extracts of Artocarpus altilis Park. and Azadirachta indica A. Juss. on the reproductive physiology of the adult female tick, Boophilus microplus (Canest.). Invertebrate Reproduction and Development, v. 23, n. 2-3, p. 159-164, 1993. 